\title{
Screening of Aloe vera medium with different carbon and nitrogen sources for Lactobacillus acidophilus cultivation using fractional factorial design (FFD)
}

\begin{abstract}
The aim of this research was to optimize the cultivation medium for economic production of a probiotic bacterium, Lactobacillus acidophilus using Aloe vera medium with different carbon (glucose, fructose and sucrose) and nitrogen (yeast extract, meat peptone, ammonium sulphate and urea) sources. Screening step was performed using $281 / 16$ fractional factorial design (FFD) to investigate the significant effect of 8 factors used in this study on the biomass production expressed in $\log 10 \mathrm{cfu} / \mathrm{mL}$. Biomass production was measured based on total plate count method for enumeration of viable cells. In the process of screening, the concentration range of Aloe vera, carbon and nitrogen used were from 1-2\% (w/v), 1-2\% $(\mathrm{w} / \mathrm{v})$ and $0.5-1 \%(\mathrm{w} / \mathrm{v})$, respectively. The maximum biomass production was obtained with $11.816 \log 10 \mathrm{cfu} / \mathrm{mL}$. It was shown that glucose, Aloe vera gel, combination of glucose and fructose and combination of glucose and ammonium sulphate were resulted significant ( $\mathrm{p}<$; 0.05 ) effect towards to the response, biomass production.
\end{abstract}

Keyword: Optimization; Aloe vera gel; Lactobacillus acidophilus; FFD 